\title{
Abhandlungen
}

\section{Andrea Hausmann}

\section{Preispolitische Optionen zur Erlösoptimierung von Museumsbetrieben}

Eigeneinnahmen; Museumsbetriebe; Preisbereitschaft; Preisbestimmung; Preisbündelung; Preisdifferenzierung; Preispolitik; Preisvariation

Die Finanzierung öffentlicher Kulturangebote ist zu einem zentralen Thema des Kulturmanagement geworden. Da die bisherigen Finanzierungsformen nur noch in eingeschränkten Umfang erhalten bleiben, müssen sich Kulturbetriebe wie z.B. die Museen verstärkt um die Ausschöpfung alternativer Finanzierungsquellen und die Erhöhung der eigenen Einnahmen bemühen. Neben der Einwerbung von privaten und öffentlichen Drittmitteln stehen Maßnahmen zur Steigerung der Erlöse im Mittelpunkt der Überlegungen. In diesem Zusammenhang spielt eine den Spezifika des Kulturbereichs gerecht werdende Ausgestaltung der Preispolitik eine wesentliche Rolle.

\section{Problemstellung}

Die Finanzierung kultureller Aufgaben erfolgt in kaum einem anderen Land in solch starkem Maße aus staatlichen Mitteln wie in Deutschland - nach wie vor werden über 90\% der Mittel für Kultur von der öffentlichen Hand aufgebracht. Aufgrund der prekären Haushaltssituation fahren Bund, Länder und Gemeinden ihre Kulturausgaben seit 2001 allerdings sukzessive zurück (vgl. Tabelle 1): Während der Kulturbereich in 2001 noch mit rund 8,4 Milliarden EUR unterstützt wurde, sanken die Ausgaben der öffentlichen Haushalte für Kunst und Kulturpflege nach den vorläufigen Ergebnissen der Haushaltsansatzstatistik des Statistischen Bundesamtes bis 2003 um knapp 2\% auf rund 8,2 Milliarden EUR. ${ }^{1}$ Auch für das Jahr 2004 ist nach Meinung von Experten von einem weiteren signifikanten Rückgang auszugehen. ${ }^{2}$

1 Vgl. Statistisches Bundesamt (2004), S. 20.

2 Vgl. Statistisches Bundesamt (2005); Söndermann (2004), S. 355. 


\begin{tabular}{|l|c|c|c|}
\hline & $\mathbf{2 0 0 1}$ & $\mathbf{2 0 0 2}$ & $\mathbf{2 0 0 3}$ \\
\hline Kulturausgaben insgesamt & & & \\
\hline Veränderung zum Vorjahr in \% & 8.355 & 8.231 & 8.193 \\
\hline
\end{tabular}

Tab. 1: Ausgaben der öffentlichen Hand für Kunst und Kulturpflege (in Mio. EUR) Quelle: $\quad$ Eigene Darstellung

Der Bereich Theater und Musik bindet dabei traditionell den größten Teil der öffentlichen Kulturausgaben. So flossen im Jahr 2001 über 3 Milliarden EUR und damit über ein Drittel (36,9\%) der gesamten Kulturausgaben von Bund, Ländern und Gemeinden in diese Sparte. Den zweiten großen Ausgabenblock bildeten in 2001 die Museen, die mit rund 1,4 Milliarden EUR unterstützt wurden (16,5\%). Weitere rund 1,4 Milliarden EUR flossen in die Finanzierung der Bibliotheken (16,4\%), die damit in 2001 den drittgrößten Ausgabenblock darstellten. Von den verbleibenden 30\% der Gesamtsumme öffentlicher Kulturausgaben wurden anteilig der Denkmalschutz und die Denkmalpflege, die kulturellen Angelegenheiten im Ausland, die Kunsthochschulen, die sonstige Kulturpflege und die Verwaltung für kulturelle Angelegenheiten gefördert (vgl. Tabelle 2). ${ }^{3}$

Im Hinblick auf die Frage, ob von dem skizzierten Rückgang der öffentlichen Kulturfinanzierung de facto alle Kulturbereiche gleichermaßen betroffen sind, zeigt die Gegenüberstellung der Ergebnisse von 2001 mit denen von 2002 und 2003 kein einheitliches Bild (vgl. Tabelle 2). Während einige Kulturbereiche ein Auf und Ab bei der Höhe der Finanzierung durch die öffentliche Hand verzeichnen, müssen andere Sparten einen kontinuierlichen und zum Teil deutlichen Rückgang hinnehmen. ${ }^{4}$

3 Vgl. Statistisches Bundesamt (2004), S. 50 ff. Mit dem Kulturfinanzbericht 2003 wurde von den kulturpolitisch Verantwortlichen erstmals ein gemeinsames Konzept zur Definition des Kulturbegriffs vorgelegt. Die oben genannten Bereiche zählen damit zu den unbestritten kulturrelevanten Aufgabenbereiche von Bund, Ländern und Gemeinden in der öffentlichen Kulturfinanzierung. Vgl. Söndermann (2004), S. 354.

Vgl. Statistisches Bundesamt (2004), S. 52 ff. 


\begin{tabular}{|c|c|c|c|c|c|}
\hline & 2001 & 2002 & $\Delta$ in $\%^{5}$ & 2003 & $\Delta$ in $\%^{6}$ \\
\hline Theater und Musik & 3.080 & 3.146 & $+2,1$ & 3.143 & $-0,1$ \\
\hline $\begin{array}{l}\text { Museen, Sammlungen und } \\
\text { Ausstellungen }\end{array}$ & 1.376 & 1.276 & $-7,2$ & 1.267 & $-0,7$ \\
\hline Bibliotheken & 1.372 & 1.349 & $-1,7$ & 1.353 & $+0,3$ \\
\hline $\begin{array}{l}\text { Denkmalschutz und } \\
\text { Denkmalpflege }\end{array}$ & 404 & 382 & $-5,4$ & 347 & $-9,2$ \\
\hline $\begin{array}{l}\text { Kulturelle Angelegenheiten im } \\
\text { Ausland }\end{array}$ & 315 & 320 & $+1,6$ & 304 & $-5,0$ \\
\hline Kunsthochschulen & 436 & 429 & $-1,6$ & 441 & $+2,8$ \\
\hline Sonstige Kulturpflege & 899 & 839 & $-6,7$ & 843 & $+0,5$ \\
\hline $\begin{array}{l}\text { Verwaltung für kulturelle } \\
\text { Angelegenheiten }\end{array}$ & 473 & 489 & $+3,4$ & 495 & $+1,2$ \\
\hline
\end{tabular}

Tab. 2: Öffentliche Ausgaben für Kultur nach Kulturbereichen (in Mio. EUR)

Quelle: $\quad$ Eigene Darstellung

Letzteres betrifft die im Rahmen der vorliegenden Fragestellung vorrangig interessierende Kultursparte der Museen, die neben dem Denkmalschutz seit 2001 den insgesamt größten Rückgang an öffentlichen Finanzmitteln verkraften muss. Dies ist für viele der über 3.400 öffentlichen Museumsbetriebe auch insofern von bedrohlicher Relevanz als rund drei Viertel der gesamten Museumsfinanzierung bereits in 40 Großstädten mit 200.000 und mehr Einwohnern gebunden werden - der breite, regional weit gefächerte Rest muss mit dem verbleibenden Anteil an der dazu noch rückläufigen Finanzierungssumme auskommen. ${ }^{7}$ Entsprechend bleiben in vielen Häusern freie Personalstellen unbesetzt und das Leistungsspektrum z.T. deutlich eingeschränkt werden. ${ }^{8}$ Summa summarum kann daher für die überwiegende Zahl der Akteure im Museumsbereich festgehalten werden, dass die finanzielle Situation in den letzten Jahren deutlich weniger komfortabel geworden ist und vielerorts mit knapperen Etats kalkuliert werden muss. Und da die öffentliche Hand wohl unbestritten vor der größten finanzpolitischen Aufgabe seit Bestehen der Bundesrepublik steht, ist ein Ende dieser Abwärtsspirale auch noch nicht abzusehen selbst wenn die Haushaltsprobleme wohl unbestritten nicht über Kürzungen in den Kulturetats bei Bund, Ländern und Gemeinden gelöst werden können. Es kann vielmehr davon ausgegangen werden, dass der finanzielle Abschmelzungsprozess in den nächsten Jahren weitergehen und v.a. jene Kulturbetriebe treffen wird, deren institutionellen und vertraglichen Strukturen gegen einen (raschen) Finanzabbau weniger resistent sind und/oder die aufgrund ihrer Ausgangssituation (z.B. kleinere Gemeinden mit geringerem Pro-Kopf-Anteil der Kulturausgaben) schneller ein Opfer der Sparmaßnahmen werden. ${ }^{9}$

\footnotetext{
Veränderung gegenüber dem Vorjahr in Prozent.

Veränderung gegenüber dem Vorjahr in Prozent.

Vgl. Söndermann (2004), S. 359 f.

Vgl. Wagner (2004), S. 21.

9 Vgl. Söndermann (2004), S. 370 f.
} 
Vor diesem Hintergrund sind viele Museen geradezu gezwungen weitere Quellen zur langfristigen Stabilisierung oder Verbesserung ihrer finanziellen Situation zu erschließen, wenn sie ihr Leistungsspektrum künftig aufrechterhalten oder sogar - in Anbetracht der zunehmenden Konkurrenz auf dem Freizeitmarkt - ausbauen wollen. Hierzu sind grundsätzlich alle Maßnahmen geeignet, die zu einer Erhöhung der

- Umsatzerlöse,

- $\quad$ sonstigen betrieblichen Erträge und

- $\quad$ öffentlichen und privaten Drittmittel

führen. Im Mittelpunkt der nachfolgenden Ausführungen stehen die Möglichkeiten der Preispolitik zur Erhöhung der Umsatzerlöse. Hierunter werden alle Einnahmen aus dem Verkauf von Waren und Dienstleistungen erfasst, die von den Museumsbetrieben im Rahmen ihres Kern- und Zusatzgeschäfts erstellt bzw. bereitgestellt werden. Von den sonstigen betrieblichen Erträgen sind im Rahmen der vorliegenden Fragestellung v.a. Einnahmen aus der Vermietung von Räumen an externe Nutzer und für Zwecke, die außerhalb der gewöhnlichen Geschäftstätigkeit stehen, sowie aus Mitgliederbeiträgen der Träger- und Fördervereine von Interesse, da Erträge, die z.B. aus Ausschüttungen aus betriebsnahen Stiftungen und aus Kapitalerträgen stammen, von Maßnahmen der Preispolitik nicht beeinflusst werden können. ${ }^{10}$

Ein Blick in die Literatur zum Stand der Forschung bei diesem für die Kultureinrichtungen so existenziellen Thema überrascht: Wenngleich verschiedene allgemein gehaltene Ansätze zur Verbesserung der finanziellen Situation für Kulturbetriebe existieren, ${ }^{11}$ so mangelt es doch an einer dezidierten Darstellung preispolitischer Optionen zur Erhöhung der Umsatzerlöse. Und das, obwohl Heinrichs schon vor fast zehn Jahren anmerkte: „Während in kommerziellen Kulturbetrieben die Erlöse aus dem Verkauf von Waren und Dienstleistungen im Mittelpunkt der Zahlungsströme stehen und i.d.R. den bei weitem größten Teil der Erträge ausmachen, finden sie in öffentlichen und gemeinnützigen Kulturbetrieben häufig nur geringe Beachtung. Zweifellos aber wird man auch hier in $\mathrm{Zu}-$ kunft den Umsatzerlösen weit größere Aufmerksamkeit schenken müssen, weil es sich um einen der wenigen Bereiche handelt den die Kulturmanager weitgehend unabhängig von außerbetrieblichen Strukturen und Entwicklungen selbst beeinflussen und gestalten können. ${ }^{12}$ Vor dem Hintergrund dieses Forschungsdefizits verwundert es nicht, dass in der Praxis vieler Museen und anderer Kultureinrichtungen erhebliche Unsicherheiten bezüglich der preispolitischen Möglichkeiten zur Erlösoptimierung bestehen. Erschwerend kommt hinzu, dass der preispolitische Handlungsspielraum öffentlicher Museen Besonderheiten aufweist und sich deutlich von dem der kommerziellen Kulturbetriebe und der anderen erwerbswirtschaftlichen Dienstleistungsbetriebe unterscheidet. ${ }^{13}$ Aus diesem Grund werden nachfolgend zunächst die Bestimmungsfaktoren der Preispolitik sowie die

10 Dies gilt gleichfalls für Maßnahmen zur Verbesserung der Drittmittelsituation (Sponsoring, Spenden, Zuwendungen aus Stiftungen etc.), die aus diesem Grund ebenfalls keine weitere Berücksichtigung finden; vgl. ausführlicher hierzu Bruhn (2003); Duda/Hausmann (2004); Hausmann (2005).

11 Vgl. Heinrichs (1998); Heinze (2002); Klein (2003).

12 Vgl. Heinrichs (1997), S. 169.

13 Zur Einordung von Kulturbetrieben als kulturelle Dienstleistungsbetriebe vgl. ausführlich Hausmann (2001); dies. (2005). 
wesentlichen Prinzipien der Preisfestlegung für Museumsleistungen skizziert, bevor anschließend Optionen zur Optimierung der Umsatzerlöse und der sonstigen betrieblichen Erträge expliziert werden. Dass sich hier in der derzeit angespannten Situation tatsächlich ein Handlungsspielraum für die Museen eröffnet, ist nicht zuletzt dem Umstand geschuldet, dass die Besuchszahlen, die für die oben genannten Erlösarten eine wichtige Rolle spielen, in 2004 um rund 5\% gegenüber dem Vorjahr gestiegen sind. ${ }^{14}$

\section{Bestimmungsfaktoren der Preispolitik von Museumsbetrieben}

Die Preispolitik befasst sich mit der Höhe der für die Leistungen von Museumsbetrieben zu entrichtenden Entgelte. Der Preis steht als Entgeltfaktor dem gesamten Leistungsbündel der Einrichtungen gegenüber. Er beeinflusst nicht nur die Entscheidung eines Besuchers, eine Museumsleistung in Anspruch zu nehmen, sondern auch die Auswahl einer Leistung innerhalb eines (kulturellen) Angebotsumfelds. Preispolitische Entscheidungen sind nicht zuletzt deshalb so bedeutsam, weil sie sowohl auf die Mengen- als auch auf die Wertkomponente der Umsatzerlöse einwirken. ${ }^{15}$ Im Allgemeinen werden preispolitische Entscheidungen getroffen, wenn es

- um die Festsetzung eines Preises für eine neue bzw. eine einmalige Leistung und/oder

- $\quad$ um Preisänderungen auf Grund von Veränderungen in Angebot und Nachfrage, von Aktionen der Wettbewerber oder von Beschlüssen der Träger geht. ${ }^{16}$

Während die Preispolitik im Marketing für erwerbswirtschaftliche Dienstleistungsbetriebe einen wesentlichen Aktionsparameter darstellt und wie kein anderes marketingpolitisches Instrument auf Absatz, Umsatz und Gewinn wirkt, verfügen die Museen über einen deutlich geringeren Gestaltungsspielraum. Im öffentlichen Museumsbereich ist als Besonderheit zu beachten, dass die Eintrittspreise, die den größten Anteil an den Umsatzerlösen von Museen ausmachen, im Regelfall nicht allein ökonomisch festgelegt werden, d.h. an den Kosten orientiert sind, sondern vielmehr (kultur-)politischen Vorgaben unterliegen und von den Rechtsträgern bzw. Eignern zumindest mitbestimmt werden. Damit können Museen unabhängig von der konkreten Trägerschaft und Rechts- bzw. Betriebsform ihre Kosten i.d.R. nicht über den Preis auf den Besucher umlegen, d.h. sich über den Markt finanzieren, sondern bleiben in hohem Maße auf (öffentliche) Zuwendungen angewiesen. ${ }^{17}$

Eine solche kulturpolitisch motivierte Festlegung von Preisen sollte allerdings in erster Linie auf den Bereich der Eintrittskarten beschränkt bleiben (und die Museumsbetriebe auch nicht daran hindern, ihre Erlöse aus dem Verkauf von Eintrittskarten durch entsprechende preispolitische Maßnahmen zu optimieren). Anders stellt sich die Situation im

14 Vgl. Institut für Museumskunde (2005), S. 7.

15 Vgl. allgemein Diller (2000), S. 14.

16 Vgl. allgemein hierzu ders. (2003), S. $11 \mathrm{f}$.

17 Vgl. Waetzold (1974), S. 160. Strachwitz 1996, S. 141 sowie ausführlich Peacock/Godfrey 1974; Jackson (1988). 
sog. Zusatz- bzw. Servicebereich ${ }^{18}$ von Kultureinrichtungen dar - hier finden sich zahlreiche Produkte und Leistungen, die unter Berücksichtigung von ökonomischen Kriterien wie z.B. marktübliches Preisniveau, Nachfrage, Wettbewerb, Deckungsbeiträge oder Kosten kalkuliert werden können. Hierzu gehören im Museumsbereich u.a.:

- $\quad$ kunstvermittelnde bzw. pädagogische Angebote (Führungen, Workshops etc.),

- $\quad$ themenbezogene (eigene) Veranstaltungen (Vorträge, Lesungen, Filmvorführungen etc.),

- Locationmanagement (Organisation und Durchführung externer Veranstaltungen),

- Produkte aus dem Museumsshop,

- Produkte der Gastronomie (falls in Eigenregie geführt),

- Vermietungs- und Verpachtungsgeschäft (inklusive Catering etc.),

- Merchandising,

- Licensing,

- Leistungen angegliederter Einrichtungen wie Bibliothek, Fotothek, Archiv, Datenbanken etc.

Grundsätzliches Ziel einer differenzierten Vorgehensweise bei der Preisbestimmung ist es im Kulturbereich, einen möglichst großen Einfluss auf die Höhe des Eigenfinanzierungsanteils der Einrichtungen zu nehmen. Wenngleich öffentliche Kulturbetriebe ihre v.a. durch Fixkosten geprägten - Kosten nicht durch eigene Einnahmen decken können (so lag z.B. der durchschnittliche Eigenfinanzierungsanteil der deutschen Bühnen in der Spielzeit 2002/03 bei rund 16\%, der von Museen bei durchschnittlich unter $25 \%)^{19}$, so ist es im Rahmen einer entsprechenden Preispolitik doch möglich, zumindest in Teilbereichen kostendeckend - in Einzelfällen sogar gewinnbringend - zu wirtschaften. Allerdings wird der Anreiz zur Steigerung der eigenen Einnahmen immer dann fehlen oder zumindest beeinträchtigt, wenn diese zusätzlich erwirtschafteten Mittel nicht dem Kulturbetrieb verbleiben, sondern in den Haushalt des Trägers fließen - ein Umstand, der durch die kameralistischen Budgetprinzipien bedingt ist und bereits seit geraumer Zeit kritisiert wird. ${ }^{20}$ Die nachfolgenden Ausführungen sind damit v.a. für jene Museumsbetriebe von Interesse, deren Rechnungslegung nach den Grundsätzen der kaufmännischen Buchführung erfolgt oder die aufgrund von Verwaltungsreformen trotz der Verpflichtung zur Kameralistik mehr Freiheit bei der Mittelverwendung haben; wie viele Häuser diese Voraussetzungen erfüllen, lässt sich zum Zeitpunkt der Analyse nicht konkret beziffern, da die statistische Datenlage im Museumsbereich in diesen Fragestellungen nach wie vor dürftig ist. Es kann allerdings davon ausgegangen werden, dass es sich hierbei um eine wachsende Zahl von Einrichtungen handelt, da immer mehr Häuser in flexiblere Rechtsformen (v.a. Stiftungen) überführt werden und die Träger zunehmend dazu übergehen, mehr Eigenverantwortung von den Häusern zu fordern.

In jedem Fall setzt die erlösoptimierende Ausgestaltung der Preispolitik von Kulturbetrieben eine genaue Kenntnis über die verschiedenen Entscheidungsphasen bei der Preis-

18 Zur Abgrenzung von Kern- und Zusatzleistungen im Kulturbetrieb vgl. Hausmann (2001); dies. (2005).

19 Vgl. Deutscher Bühnenverein (2004), S. 3. Für den Museumsbereich gibt es hierzu keine offiziellen Zahlen; die Autorin geht davon aus dass der Eigenfinanzierungsanteil bei Museen etwas höher liegt (20-25\%).

20 Vgl. Pommerehne/Frey (1993), S. 12 f.; Klein (1994), S. 24 ff.; Strachwitz (1996), S. 135 ff. 
festlegung voraus; diese sollen im Folgenden unter Berücksichtigung der besonderen Belange des Museumsbereichs näher betrachtet werden.

\section{Preispolitischer Entscheidungsprozess und museums- betriebstypische Besonderheiten}

Am Beginn des Entscheidungsprozesses steht grundsätzlich eine Analyse der IstSituation, die Aufschluss über den preispolitischen Spielraum eines Museumsbetriebs gibt. Hierbei sind u.a. zu berücksichtigen: der Organisationszweck, die Positionierung und das Angebotsprofil des eigenen Hauses, die Besucherzahlen und die Besucherstruktur, der Standort, die Wettbewerbssituation und die Vorgaben von Seiten der Träger.

Im Anschluss an diese grundlegende Analyse sind die Ziele zu formulieren, die mit der Preisfestlegung verfolgt werden. Hierbei ist zu unterscheiden zwischen museumsbetriebsbezogenen und markt- bzw. besucherorientierten Zielsetzungen; exemplarisch lassen sich nennen:

- Förderung der Einführung neuer Produkte und Dienstleistungen,

- Erschließung der Leistungen für bestimmte Zielgruppen,

- Veränderung des Images („Imagewirkung des Preises“),

- Verbesserung der Zufriedenheit mit dem Preis (,Preiszufriedenheit“)

- $\quad$ Steigerung der Besucherbindung,

- Beitrag zur Kostendeckung bzw. Verlustminimierung,

- Abschöpfung von Preisbereitschaften,

- Aufbau von Barrieren (z.B. zur Sicherstellung von Exklusivität). ${ }^{21}$

In der anschließenden dritten Phase des Planungsprozesses geht es um die Festlegung einer der jeweiligen Entscheidungssituation entsprechenden Preisstrategie; anders als bei erwerbswirtschaftlichen Dienstleistungsbetrieben verfügen die öffentlichen Museumsbetriebe in diesem Bereich jedoch nur über eingeschränkte Möglichkeiten. So kann z.B. bei attraktiven Sonderausstellungen von Museen eine Hochpreis- oder Abschöpfungsstrategie verfolgt werden; auch kann es sinnvoll sein bestimmte Produkte aus dem Museumsshop zu Promotionspreisen anzubieten oder den Einstieg in das Vermietungsgeschäft mit Hilfe einer Penetrationsstrategie zu erleichtern. Insgesamt aber lässt sich nicht verkennen, dass die meisten Strategieoptionen der Preispolitik - wie etwa Strategien des Preiswettbewerbs oder der Preisdynamik - aufgrund der Wettbewerbs- und Angebotssituation im öffentlichen Museumsbereich keine sinnvolle Anwendung finden können.

Der nächste Schritt besteht in der Auswahl einer adäquaten Methode der Preisfestlegung - auch in dieser Phase kommen die Charakteristika des Museumsbereichs nachhaltig zum Tragen. Ganz allgemein lassen sich die Methode der kostenorientierten Preisfestlegung (die über eine ,inside-out"-Perspektive verfügt) und die Methode der marktorientierten Preisfestlegung (deren Perspektive entsprechend „outside-in“ ausgerichtet ist) unter-

21 Vgl. allgemein Meffert/Bruhn (2003), S. 522. 
scheiden.$^{22}$ Letztere Verfahrensweise lässt sich weiter aufschlüsseln in die besucher- und in die konkurrenzbezogene Preisbestimmung. Die drei Methoden werden nachfolgend unter Berücksichtigung der spezifischen Belange von Museumsbetrieben skizziert:

(1) Kostenorientierte Preisbestimmung: Im Rahmen der kostenorientierten Preisbestimmung wird grundsätzlich das Ziel verfolgt, mit den Entgelten die Kosten der Leistungserstellung zu decken. Dabei zeigt sich im Dienstleistungsbereich die Problematik, dass die Aufrechterhaltung der Leistungsbereitschaft des Dienstleistungsanbieters zu einem hohen Anteil von nicht oder nur wenig beeinflussbaren Kosten (Personalkosten, Energiekosten etc.) führt; diese fixkostenlastige Struktur verhindert eine verursachungsgerechte Verteilung auf einzelne Kostenträger und erschwert die kostenorientierte Preisfestlegung. ${ }^{23} \mathrm{Ne}-$ ben diesem grundsätzlichen Problem im Dienstleistungsmarketing ist es für die Museen, aber auch für die anderen öffentlichen Kulturbetriebe aus kulturpolitischen Gründen im Regelfall nicht möglich, an den Gesamtkosten orientierte Eintrittspreise zu erheben; dies verdeutlicht ein Beispiel aus dem Theaterbereich: So bezahlte der Besucher in der Spielzeit 2002/2003 durchschnittlich 14 EUR für eine Eintrittskarte eines deutschen Stadtoder Staatstheaters; bei jeder verkauften Karte legten die Träger 95 EUR dazu - d.h. ein die Gesamtkosten deckender Eintrittspreis würde rund 110 EUR betragen. ${ }^{24}$ Es ist offenkundig, dass eine solche Entgeltforderung sowohl aus kulturpolitischen Gründen als auch aufgrund der Nachfrage- und Wettbewerbssituation nicht durchsetzbar ist. Dies bedeutet aber nicht, dass eine kostenorientierte Preisbestimmung im Kulturbereich grundsätzlich nicht möglich ist; wie bereits ausgeführt können die Kosten der Leistungserstellung v.a. im Servicebereich von Museen (Führungen, Workshops, Fachberatung, Vorträge, Vermietungsgeschäft etc.) und in Einzelfällen auch bei ausgewählten, durch hohe Besuchernachfrage gekennzeichneten Sonderausstellungen von Museen durch eine entsprechende Preispolitik gedeckt werden (als ein absolutes Ausnahmebeispiel aus der jüngsten Vergangenheit sei hier die Sonderausstellung „Das MoMa in Berlin“ der Neuen Nationalgalerie erwähnt, bei der aufgrund des überwältigenden Besucherinteresses ein Überschuss von 6,5 Mio. EUR erzielt wurde). ${ }^{25}$

(2) Besucherorientierte Preisbestimmung: Bei dieser Strategie ist die Preisbereitschaft der Besucher maßgeblich für die Festsetzung von Leistungsentgelten. Die Preis- bzw. Zahlungsbereitschaft bezieht sich auf die grundsätzliche Bereitwilligkeit eines Nachfragers, in einer Kaufsituation für eine Leistung einen bestimmten maximalen Betrag zu zahlen. Gemäß dieser Begriffsabgrenzung stellt die Preisbereitschaft die individuelle Preisobergrenze dar; Preise oberhalb der persönlichen Preisbereitschaft werden vom Nachfrager i.d.R. nicht akzeptiert. Da der maximale Preis, den ein Konsument zu zahlen bereit ist, unmittelbar mit dem wahrgenommenen Wert einer Leistung korrespondiert, kann die Preisbereitschaft entsprechend als monetärer Ausdruck des wahrgenommen Werts einer Leistung interpretiert werden. ${ }^{26}$

22 Vgl. ebd., S. 524.

23 Vgl. Meffert/Bruhn (2003), S. 517.

24 Vgl. Deutscher Bühnenverein (2004), S. $180 \mathrm{ff}$.

25 Vgl. ZDF (5. August 2005), http://www.heute.de/ZDFheute.

26 Vgl. Diller (2000), S. 168; Balderjahn (2003), S. 389. 
Die Kenntnis der Preisbereitschaft von Besuchern stellt auch im Kulturbereich eine Schlüsselinformation zur erlösoptimierenden Preisgestaltung dar. Hieraus lassen sich Preisreaktions- bzw. Preiswirkungsfunktionen (z.B. Preis-Absatz-Funktionen) ableiten, die für die Entgeltfestlegung wesentlich sind. ${ }^{27}$ Bei der Ermittlung der Preisbereitschaften muss allerdings einem grundsätzlichen Problem im Dienstleistungsbereich Rechnung getragen werden: Der Besucher kann das Preis-Leistungs-Verhältnis ohne die tatsächliche Inanspruchnahme der Leistungen aufgrund ihres überwiegend immateriellen Charakters nur unter großer Unsicherheit beurteilen. Dementsprechend sollte ihm diese Beurteilung erst nach dem Besuch einer kulturellen Veranstaltung oder der Nutzung von kulturellen Leistungen abverlangt werden. Hierzu können verschiedene Methoden eingesetzt werden, die die Preisbereitschaft entweder auf individueller Ebene (direkte Besucherbefragung, Conjoint-Analyse etc.) oder auf aggregiertem, gruppenspezifischem Niveau erfassen. ${ }^{28}$

Mit Blick auf das Ziel einer erlösoptimierenden Preisgestaltung ist darüber hinaus die Preiselastizität der Nachfrage, d.h. die Reaktion der Nachfrage auf Preisänderungen von Interesse. In empirischen Untersuchungen konnte herausgefunden werden, dass die Preiselastizität im Kulturbereich relativ gering ist. ${ }^{29}$ So führt die (moderate) Erhöhung von Eintrittspreisen i.d.R. nur zu unterproportionalen Veränderungen in der Nachfrage (d.h. $\mathrm{zu}$ einem relativ geringen Rückgang der Besucherzahlen). ${ }^{30}$ Die Gründe liegen nicht zuletzt darin, dass die Besucher von kulturellen Leistungen - insbesondere der so genannten Hochkultur - über dem Altersdurchschnitt der Bevölkerung liegen und über ein vergleichsweise höheres Einkommen verfügen, so dass höhere Eintrittspreise i.d.R. keine Besuchsbarriere darstellen.

Neben diesen Unterschieden bezüglich der Preisbereitschaft und der Preiselastizität lässt sich auf der Basis einer umfassenden Studie aus der deutschen Museumslandschaft festhalten, dass Besucher aus dem Ort eines Museums oder dem näheren Umland, Ermäßigungsberechtigte, Wiederholungsbesucher und Besucher mit einer relativ geringen Aufenthalts- und Nutzungsdauer eine geringere Preisbereitschaft und eine höhere Preiselastizität haben als Besucher von auswärts, reguläre Normalzahler, Erstbesucher und Besucher mit längeren Aufenthalts- und Nutzungsdauern (dabei liegt die Preisakzeptanz von Besuchern aus dem Ausland über der Preisakzeptanz von auswärtigen Besuchern aus dem Inland). ${ }^{31}$ Im Hinblick auf das Ziel der Erlösoptimierung ist es vor diesem Hintergrund für jeden Museumsbetrieb wichtig, die Besucher und ihre strukturellen Merkmale möglichst genau zu kennen.

(3) Konkurrenzorientierte Preisbestimmung: Diese Strategie orientiert sich bei der Preisfestsetzung an externen Kalkülen; sie setzt den Preis nach Analyse der Preise für Konkurrenzangebote (vergleichbare Kultur- und Freizeiteinrichtungen) fest. So zeigt sich z.B.,

27 Vgl. hierzu ausführlicher Balderjahn (1993), S. $40 \mathrm{ff}$.

28 Vgl. zu diesen Methoden im Einzelnen ders. (2003), S. 391 ff.

29 Vgl. Throsby/Withers (1979), S. 29; Frey/Pommerehne (1989), S. 9.

30 Diese geringe Preiselastizität der Nachfrage führt andererseits auch dazu, dass die Senkung von Eintrittspreisen i.d.R. keinen großen Einfluss auf die Gewinnung neuer Besucher bzw. die Erhöhung der Besuchszahlen hat; vgl. ausführlicher Giller (1995), S. 58 ff.; Klein (2001), S. 354 ff.

31 Vgl. Institut für Museumskunde (1996), S. 11 ff. 
dass in vielen „Block Buster“-Ausstellungen von Kunstmuseen, die z.B. aufgrund der Reputation der Künstler (Van Gogh in der Kunsthalle Bremen, Picasso in der Staatsgalerie Stuttgart etc.), des Ausstellungssujets (Tutanchamun in der Bundeskunsthalle Bonn) oder der leihenden Institutionen (Museum of Modern Art etc.) von besonderem Interesse sind, ein Eintrittspreis von rund 10 EUR gefordert wird. Ähnliches ist z.B. auch bei den Jahreskarten zu konstatieren, deren Preis in vielen Häusern - trotz unterschiedlicher Gattung, Größe und Angebotsvielfalt sowie zum Teil auch unterschiedlichem regulärem Eintrittspreis - bei 30 EUR liegt (vgl. Tab. 3). Als grundsätzliche Problematik ist bei einer allein konkurrenzorientierten Preisbestimmung jedoch zu bedenken, dass u.U. weder die Kostensituation noch die Preisbereitschaften der Nachfrager hinreichend berücksichtig werden.

Nicht zuletzt gehört die Kontrolle der eingeleiteten Maßnahmen als abschließende Aktivität zum Entscheidungsprozess der Preispolitik. Nach einer gewissen Zeit müssen die Wirkungen der preispolitischen Maßnahmen überprüft werden. Hierüber können z.B. Verkaufszahlen und Besucherstatistiken Auskunft geben. Weiterführende Informationen können im Rahmen von quantitativen oder qualitativen Besucherbefragungen gewonnen werden. Allerdings wird eine wirksame Kontrolle der preispolitischen Aktivitäten durch die möglicherweise von anderen Instrumenten des Kulturmarketing oder von Marketingmaßnahmen anderer Marktakteure (Wettbewerber, Kooperationspartner etc.) ausgehenden Wirkungen erschwert.

\begin{tabular}{|l|c|c|c|c|c|c|c|}
\hline & $\begin{array}{c}\text { MARTa } \\
\text { Herford }\end{array}$ & $\begin{array}{c}\text { Jüdisches } \\
\text { Museum } \\
\text { Berlin }\end{array}$ & $\begin{array}{c}\text { Museum für } \\
\text { Völkerkunde } \\
\text { Hamburg }\end{array}$ & $\begin{array}{c}\text { Historisches } \\
\text { Museum der } \\
\text { Pfalz, } \\
\text { Speyer }\end{array}$ & $\begin{array}{c}\text { Atlantis } \\
\text { Kinder- } \\
\text { museum, } \\
\text { Duisburg }\end{array}$ & $\begin{array}{c}\text { Bayerische } \\
\text { Schlösser- } \\
\text { verwaltung }\end{array}$ & $\begin{array}{c}\text { Stiftung } \\
\text { Weimarer } \\
\text { Klassik und } \\
\text { Kunst- } \\
\text { sammlungen }\end{array}$ \\
\hline $\begin{array}{l}\text { Jahreskarte } \\
\text { (regulär) }\end{array}$ & 30 EUR & 30 EUR & 30 EUR & 30 EUR & 30 EUR & 30 EUR & 30 EUR \\
\hline $\begin{array}{l}\text { Eintrittspreis } \\
\text { (regulär) }\end{array}$ & 6 EUR & 5 EUR & 6 EUR & 7 EUR & 7,50 EUR & $3-7$ EUR & $2-6$ EUR \\
\hline
\end{tabular}

Tab. 3: Preise für Jahreskarten ausgewählter Museen

Quelle: $\quad$ Eigene Darstellung

Im Anschluss an die verschiedenen Entscheidungsphasen der Preispolitik und ihren museumsbetriebsspezifischen Besonderheiten werden nachfolgend preispolitische Instrumente vorgestellt, deren Einsatz zu einer Optimierung der Umsatzerlöse und der sonstigen betrieblichen Erträge von Museumsbetrieben beitragen können. Dabei wird deutlich, dass außer dem Instrument der Preisdifferenzierung, dem im Museumsbereich nicht nur aus Gründen der Erlösoptimierung, sondern auch aufgrund der kulturpolitischen Maßgabe, einem möglichst großen Kreis von Personen einen Kulturbesuch zu ermöglichen und Zugangsbarrieren für bestimmte Bevölkerungsgruppen zu senken („Kultur für alle“), eine große Rolle zukommt, nur einige wenige von denen dem erwerbswirtschaftlichen Dienst- 
leistungsbereich zur Verfügung stehenden Instrumenten auch für den Museumsbereich geeignet sind.

\section{Instrumente der Preispolitik zur Erlösoptimierung von Museumsbetrieben}

\section{Preisdifferenzierung}

Im Kern geht es bei der Preisdifferenzierung um die Ausnutzung des Umstandes, dass Nachfrager auf einem Markt üblicherweise unterschiedlich hohe Zahlungsbereitschaften für die gleiche Leistung aufweisen. Vorrangiges Ziel der Preisdifferenzierung ist es auch im Dienstleistungsbereich, Konsumentenrenten abzuschöpfen und die Erlöse - und damit im Kulturbereich den Eigenfinanzierungsanteil - zu erhöhen; darüber hinaus soll durch die gezielte Beeinflussung des Nachfragerverhaltens eine gleichmäßigere Auslastung der Dienstleistungskapazitäten erreicht und die Entstehung von Leerkosten soweit wie möglich vermieden werden. ${ }^{32}$

Im Museumsbereich wird das Instrument der Preisdifferenzierung v.a. im Rahmen der Eintrittspreisgestaltung eingesetzt. ${ }^{33}$ Durch Preisdifferenzierung setzt sich der Erlös aus dem Verkauf von Eintrittskarten wie folgt zusammen:

$$
\mathrm{E}_{\text {Museum }}=\left(\mathrm{p}_{1} * \mathrm{x}_{1}\right)+\left(\mathrm{p}_{2} * \mathrm{x}_{2}\right)+\left(\mathrm{p}_{3} * \mathrm{x}_{3}\right)+\ldots .+\left(\mathrm{p}_{\mathrm{n}} * \mathrm{x}_{\mathrm{n}}\right)
$$

Dabei können $\mathrm{p}_{1}$ bzw. $\mathrm{x}_{1}$ der reguläre Eintrittspreis bzw. die Anzahl der verkauften Eintrittskarten an Vollzahler, $\mathrm{p}_{2}$ bzw. $\mathrm{x}_{2}$ der ermäßigte Eintrittspreis für Studierende bzw. die Anzahl der verkauften Eintrittskarten an Studierende, $\mathrm{p}_{3}$ bzw. $\mathrm{x}_{3}$ der Eintrittspreis für andere Ermäßigungsberechtigte (Familien, Gruppenbesucher, Jahreskarteninhaber etc.) bzw. die Anzahl der verkauften Eintrittskarten an Ermäßigungsberechtigte etc. sein. Es ist offenkundig, dass der Gesamterlös eines Museums steigt, wenn es durch Maßnahmen der Preisdifferenzierung gelingt, Besucher mit höherer Preisbereitschaft zu entsprechend höheren Preisen zu bedienen und Besucher mit geringerer Preisbereitschaft, die ansonsten auf den Besuch des Museums bzw. die Inanspruchnahme seiner Leistungen verzichten würden, durch einen entsprechend ermäßigten Preis zu gewinnen. ${ }^{34}$

Zur Differenzierung von Preisen für kulturelle Leistungen können folgende Kriterien isoliert oder kombiniert - herangezogen werden.

32 Vgl. Diller (2000), S. 294 ff.; Meffert/Bruhn (2003), S. 529; Fassnacht (2003), S. 485 ff.

33 Darüber hinaus wird das Instrument der Preisdifferenzierung z.B. auch im Rahmen der Entgeltfestsetzung für pädagogische Leistungen, Raumvermietungen, Nutzungen sonstiger Einrichtungen eines Museums oder Mitgliedschaften in Förder- bzw. Trägervereinen eingesetzt.

34 Vgl. allgemein Diller (2000), S. 294. 


\section{a) Räumliche Preisdifferenzierung}

Im Rahmen dieser Strategie werden preisliche Differenzierungen zwischen den verschiedenen Ausstellungsräumen bzw. Standorten eines Museums vorgenommen. So beträgt etwa der reguläre Eintrittspreis bei den Staatlichen Museen zu Berlin für den außerhalb gelegenen und weniger frequentierten Museumsstandort „Dahlem“ 4 EUR und für den zentral gelegenen und besonders beliebten Museumsstandort „Museumsinsel“ 8 EUR. Diese Form der Preisdifferenzierung bleibt allerdings jenen Museen und Museumskomplexen vorbehalten, die über verschiedene Einrichtungen bzw. Stätten verfügen, wie neben den Staatlichen Museen zu Berlin z.B. auch die Staatlichen Kunstsammlungen Dresden, die Stiftung Weimarer Kunst und Klassik oder die Stiftung Preußische Schlösser und Gärten. Museen mit ausschließlich einem Standort können diese Form der Preisdifferenzierung zwangsläufig nicht im Bereich der Eintrittspreisgestaltung anwenden, zu prüfen ist aber z.B., ob im Rahmen des Vermietungsgeschäfts unterschiedliche Räume unter Berücksichtigung geeigneter Kriterien (Größe, Ausstattung, Atmosphäre etc.) zu unterschiedlichen Preisen vermietet werden können.

\section{b) Zeitliche Preisdifferenzierung}

Bei dieser Form der Preisdifferenzierung ist der Zeitpunkt der Inanspruchnahme von kulturellen Leistungen ausschlaggebend. So lässt sich im Museumsbereich als Beispiel die so genannte „Happy Hour“ anführen, zu der die Häuser eine Stunde vor der regulären Museumsschließung vergünstigt (z.B. in der Kunsthalle Hamburg für 3 EUR gegenüber einem regulärem Eintrittspreis von 8,50 EUR) besucht werden können. Diese Möglichkeit zur Einnahmegenerierung - jede nicht verkaufte Eintrittskarte stellt letztlich eine entgangene Einnahme dar - wird von vielen Museen allerdings noch zu wenig ausgeschöpft; häufig genug bleibt das Angebot aufgrund fehlender kommunikationspolitischer Flankierung den (potenziellen) Nutzern (v.a. Wiederholungs- und Stammbesucher, aber auch Kulturtouristen) unbekannt und ungenutzt. Diese Form der Preisdifferenzierung eignet sich darüber hinaus auch für andere entgeltpflichtige Aktivitäten von Museen, wie z.B. die Raumvermietung (unterschiedliche Preise für Anmietung wochentags oder am Wochenende) oder die Inanspruchnahme pädagogischer Angebote (Führungen unter der Woche und am Wochenende etc.).

\section{c) Besucherorientierte Preisdifferenzierung}

Diese Variante der Preisdifferenzierung knüpft an die mit verschiedenen persönlichen Merkmalen (Alter, soziale Stellung, Besucherstatus, Familienstand etc.) variierende Preisbereitschaft der Besucher bei der Inanspruchnahme von kulturellen Leistungen an. Eine solche Preisdifferenzierung nach besucherbezogenen Merkmalen nimmt die Mehrheit der knapp dreieinhalbtausend öffentlichen Museen in Deutschland vor, hierbei zei- 
gen sich allerdings deutliche Unterschiede in der Art und dem Umfang der begünstigten Personenkreise (vgl. Tabelle 4) sowie auch im Ausmaß des Preisnachlasses. So beträgt die Ermäßigung in manchen Häusern bis zu 50\% (z.B. Kunsthalle Bremen, Deutsches Technikmuseum Berlin), in anderen liegt sie mit 25 - 30\% deutlich darunter (z.B. Kunstsammlung NRW, Deutsche Arbeitsschutzausstellung Dortmund). Mit Blick auf das Ziel der Erlösoptimierung sollte in nicht wenigen Museen kritisch darüber nachgedacht werden, ob Umfang und Ausmaß der besucherorientierten Preisdifferenzierung zumindest in Teilen eingeschränkt werden können, ohne dass der gesellschaftspolitische Auftrag gefährdet wird.

\begin{tabular}{|l|c|}
\hline Personenkreise mit Ermäßigung & $\begin{array}{c}\text { Anzahl der Museen mit } \\
\text { ermäßigtem Eintritt (in \%) }\end{array}$ \\
\hline Kinder & 46,2 \\
\hline Jugendliche/Schüler & 74,2 \\
\hline Studenten & 64,4 \\
\hline Behinderte & 55,8 \\
\hline Auszubildende & 48,7 \\
\hline Rentner & 29,3 \\
\hline Sozialhilfeempfänger & 37,4 \\
\hline Erwachsenengruppen & 59,2 \\
\hline andere Gruppen & 9,4 \\
\hline einheimische Schulklassen & 38,8 \\
\hline andere Schulklassen & 49,1 \\
\hline Familienkarte & 34,6 \\
\hline Fördervereinsmitglieder & 2,8 \\
\hline Mitglieder des Deutschen Museumsbunds & 1,9 \\
\hline Mitglieder des International Council of Museums & 2,1 \\
\hline Sonstige & 12,3 \\
\hline
\end{tabular}

Tab. 4: Personenkreise differenziert nach ermäßigtem und freien Eintritt

Quelle: $\quad$ Institut für Museumskunde (2005), S. 36

\section{d) Mengenorientierte Preisdifferenzierung}

Diese Form der Preisdifferenzierung wird in Abhängigkeit von der Anzahl der bei einem Kulturbetrieb nachgefragten Dienstleistungseinheiten vorgenommen. Im Museumsbereich spielen hier v.a. die Jahreskarten eine Rolle. Mit dem Kauf einer Jahreskarte ist der Besucher berechtigt, ein bestimmtes Museum ohne weitere Entgeltentrichtung innerhalb eines Jahres unbegrenzt häufig zu besuchen; besonders erlösoptimierend denkende Häuser bieten dabei zwei Varianten an, die dem Nutzungsverhalten unterschiedlicher Besuchertypen gerecht werden (Variante 1: Freier Eintritt begrenzt auf die Dauerausstellung, Variante 2: Freier Eintritt für Dauer- und Sonderausstellungen). Der Vorteil solcher Jah- 
reskarten liegt für die Museen in den „sicheren“ Einnahmen: Der Besucher zahlt zu einem bestimmten Zeitpunkt den veranschlagten Preis, unabhängig von seinem tatsächlichen Nutzungsverhalten im Jahresverlauf.

Die durch Preisdifferenzierung erhofften Erlöseffekte werden allerdings nur dann eintreten, wenn die Besucher bzw. Nutzer eines Museums de facto unterschiedliche Preisbereitschaften und Preiselastizitäten aufweisen und sich die zu diesem Zweck gebildeten Besuchersegmente zumindest in einem gewissen Ausmaß voneinander unterscheiden. ${ }^{35}$ Darüber hinaus ist zu vermeiden, dass die Übersichtlichkeit des Preissystems eines $\mathrm{Mu}-$ seumsbetriebs durch eine übermäßige Ausweitung der Preisdifferenzierung gefährdet wird; auch kann es durch den Einsatz mehrerer Varianten der Preisdifferenzierung zu Überschneidungen kommen, die zu einer Unzufriedenheit bei dem Besucher führen kann. ${ }^{36}$

\section{Preisvariation}

Preisvariationen liegen vor, wenn ein Museum den Preis für bestimmte Leistungen innerhalb eines bestimmten - kurz- oder längerfristigen - Zeitraums zur bewussten Beeinflussung des Marktes verändert, d.h. anhebt oder absenkt. Kurzfristige Preisaktionen (,Sonderangebote") im Museumsshop oder bei der Vermietung von Räumen können die Nachfrage stimulieren und zu einer Erhöhung der Erlöse beitragen. Von der zeitlichen Preisdifferenzierung unterscheidet sich die Preisvariation durch ihre Unregelmäßigkeit und aus der Perspektive der Besucher - Unvorhersehbarkeit, zudem gelten bei Preisvariationen keine unterschiedlichen Preise parallel.$^{37}$ Neben Preisabschriften, d.h. Reduktionen eines ursprünglich geplanten, aber nicht mehr durchsetzbaren Verkaufspreises (z.B. für den Katalog einer abgelaufenen Sonderausstellung oder für Merchandisingartikel im Shop), gilt als weitere Form der Preisvariation das so genannte Yield Management, das im Kulturbereich v.a. von Musiktheatern und Musicals mit hohem Besucherzuspruch eingesetzt wird, in relativ selten Fällen aber auch von Museen mit hochkarätigen Sonderausstellungen, weswegen dieses Instrument der Vollständigkeit halber kurz vorgestellt werden soll. Das Yield Management beinhaltet eine Weiterentwicklung des Gedankens der zeitlichen Preisdifferenzierung, in deren Mittelpunkt eine ertragsorientierte Steuerung der Angebotsmengen von Dienstleistungen, die - wie bei Theaterinszenierungen, Opernaufführungen, Konzerten und „Block“ Buster-Ausstellungen - im Voraus buchbar sind und an unterschiedlich preissensitive Besuchergruppen vertrieben werden, durch optimale Auslastung gegebener Kapazitäten steht. ${ }^{38}$ So konnten bspw. im Rahmen der „MoMa“Ausstellung im Vorfeld Karten für so genannte ,time slots“ bestellt werden, die dem Besucher das Anstehen in der langen Schlange vor dem Museum ersparten und ihm zu einer bestimmten Zeit für eine bestimmte Zeitspanne Einlass in die Ausstellung gewährten; der

35 Vgl. Fassnacht (2003), S. 487.

36 So ist es z.B. im Museumsbereich häufiger anzutreffen, dass sich die Leistungen von Kombitickets, Tages- und Sammelkarten überschneiden.

37 Vgl. allgemein Diller (2000), S. 325.

38 Vgl. allgemein Diller (2000), S. 461; Pechtl (2005), S. 250 ff. 
Preis für diese Karten lag deutlich über dem regulären Eintrittspreis und richtete sich dementsprechend an wenig preissensitive Kulturnutzer.

\section{Preisbündelung}

Als Preisbündelung wird die Zusammenstellung mehrerer identifizierbarer Teilleistungen (Produkte, Dienstleistungen und/oder Rechte) eines Anbieters oder mehrerer Anbieter zu einem Angebotsbündel mit Ausweis eines Gesamtpreises bezeichnet, wobei der Gesamtpreis unter der Summe der Einzelpreise liegt. ${ }^{39}$ Im Rahmen der Preispolitik von erwerbswirtschaftlichen Dienstleistungsbetrieben bietet die Preisbündelung, die als ein Spezialfall der leistungs- bzw. mengenbezogenen Preisdifferenzierung gesehen werden kann, oft erhebliche Potenziale zur Ertragssteigerung, v.a. durch die Möglichkeit einer stärkeren Abschöpfung von Konsumentenrenten. Voraussetzung hierfür sind v.a. eine heterogene Nachfrage, die Entbündelbarkeit bisheriger oder die Bündelbarkeit vorhandener Teilleistungen sowie ein fehlender Wettbewerb mit Spezialanbietern für Teilleistungen. ${ }^{40}$

Auch im Museumsbereich kann der Einsatz der Preisbündelung dazu beitragen, die Umsatzerlöse und die sonstigen betrieblichen Erträge zu erhöhen. Von den verschiedenen Gestaltungsvarianten wählen Museen i.d.R. eine reine und/oder eine gemischte Preisbündelung (,pure bundling“ bzw. „mixed bundling“). Im Rahmen der reinen Preisbündelung offeriert das Museum die einzelnen Leistungen des Angebotsbündels als Komplettpaket, da die Leistungen nicht einzeln erhältlich sind bzw. keine Einzelpreise ausgewiesen werden (können). ${ }^{41}$ Beispiele hierfür sind die Bündelung verschiedener Einrichtungen bzw. Standorte eines Museums im Rahmen einer so genannten Gemeinschaftskarte, wobei es für bestimmte Häuser kein Einzelticket gibt (wie etwa bei der Kunsthalle Bremen für das Gerhard-Marcks-Haus und das Wilhelm Wagenfeld Haus) ${ }^{42}$, oder die Bündelung verschiedener attraktiver Leistungen, die im Rahmen des Beitritts zum Förderverein oder Freundeskreis von Kulturbetrieben bzw. gegen Zahlung des Mitgliedsbeitrags erworben werden können (und deren Leistungsbestandteile, wie z.B. Ausstellungsführungen oder Atelierbesuche, nicht einzeln erhältlich sind).

Im Rahmen der gemischten Preisbündelung bieten Museen neben einem Gesamtpaket mit dem Bündelpreis auch alle Teilleistungen zu Einzelpreisen an. Diese Form wird auch „optional bundling“ genannt, weil dem Besucher die Entscheidung zwischen dem Kauf einer Einzelleistung und dem Leistungsbündel freigestellt wird: Einem Besucher, der zum Erwerb des gesamten Bündels nicht bereit ist (aus finanziellen Gründen, Zeitgründen etc.), bleibt bei dieser Variante der Preisbündelung die Möglichkeit erhalten zumindest eine Teilleistung in Anspruch zu nehmen. Wiederum kommt ein anderer Besucher beim Bündelkauf z.B. in den Genuss zusätzlicher Vorteile (i.d.R. wird der Kauf des Leis-

39 Vgl. Diller (2003), S. 14; Priemer (2003), S. 506 ff.

40 Vgl. Simon (1992), S. 442 ff.; Diller (2000), S. 306.

41 Vgl. allgemein Pechtl (2005), S. 168.

42 Ziel einer solchen Bündelung ist es häufig, besucherschwächere Einrichtungen mit geringerem Bekanntheitsgrad im „Fahrwasser“ besucherstarker, über einen hohen Bekanntsheitsgrad verfügender Einrichtungen mitzuziehen. 
tungsbündels durch einen Preisvorteil gegenüber dem Einzelkauf honoriert). ${ }^{43}$ Beispiele für gemischte Preisbündelungen im Museumsbereich, die häufig die Kooperation zwischen verschiedenen Kulturbetrieben bzw. mit erwerbswirtschaftlichen Dienstleistungsbetrieben (Hotellerie etc.) erfordern, sind so genannte Kombitickets von Museen, in deren Rahmen verschiedene, z.B. thematisch zusammengehörende Einrichtungen besucht werden können (so berechtigt das „MQ Art Ticket“ des Museumsquartiers Wien für 25 EUR zum Eintritt in drei Kunstmuseen, die jeweils auch gegen Kauf eines Einzeltickets besucht werden können). ${ }^{44}$ Ähnliches gilt für die aufgrund des dynamischen Wachstums des Marktsegments Kulturtourismus mehr und mehr von Kulturbetrieben geschnürten kulturtouristischen Leistungspakete, in deren Rahmen z.B. Übernachtungspauschalen sowie die Eintrittskarten und die Führung durch die Ausstellungen zu einem Paketpreis angeboten werden.

\section{Fazit}

Den öffentlichen Museen stehen verschiedene preispolitische Optionen zur Optimierung ihrer Erlöse zur Verfügung. Hierzu geeignet sind v.a. Maßnahmen der Preisdifferenzierung sowie in deutlich eingeschränktem Umfang auch Maßnahmen der Preisvariation und der Preisbündelung. Dabei gibt es keine allgemeingültigen Entscheidungsregeln für oder gegen den Einsatz eines bestimmten preispolitischen Instruments. Vielmehr muss jedes Museum die Auswahl geeigneter Optionen unter Berücksichtigung seiner ganz konkreten Ausgangssituation treffen (Besucherstruktur, Leistungsspektrum etc.). Grundsätzlich sollten aber verschiedene Parameter der Preispolitik bzw. museumsbetriebsspezifischen Charakteristika bei der Entscheidungsfindung im Blick behalten werden; hierzu gehören u.a.:

- Maßnahmen der Preispolitik sind mit anderen Instrumenten des Museumsmarketing (Kommunikationspolitik etc.) abzustimmen, damit es nicht zu konfliktären Wirkungsverhältnissen kommt.

- Maßnahmen der Preisbündelung können kurzfristig die Nachfrage erhöhen, langfristig jedoch zu einer Reduktion der Ge- und Verbrauchsintensität führen und sich damit u.U. negativ auf die Besucherbindung auswirken. ${ }^{45}$

- Bei der Preisbündelung im Rahmen von Kombitickets, Besucherpässen etc. kann es $\mathrm{zu}$ Vermarktungsschwierigkeiten und Abgrenzungsproblemen bei der Aufteilung der Erlöse zwischen verschiedenen Einrichtungen kommen.

- Überschreiten die Erlöse aus dem wirtschaftlichem Geschäftsbetrieb einen bestimmten Betrag, verliert das Museum unter Umständen die Anerkennung der Gemeinnützigkeit (und damit die Befreiung von der Körperschaftsteuer).

43 Vgl. allgemein Priemer (2003), S. 507; Pechtl (2005), S. 168.

44 Vgl. Museumsquartier (9. August 2005), http://www.museumsquartier.at.

45 Dies ist dem Umstand geschuldet, dass die Loslösung der Zahlung vom Leistungserlebnis zu einer reduzierten Bereitschaft führen kann, diese Leistung auch tatsächlich in Anspruch zu nehmen. Herrmann verdeutlicht dies anhand eines empirischen Beispiels aus dem Musikfestivalbereich; vgl. Herrmann (2003), S. 41 f. 
Diese Aspekte lassen sich durch eine sorgfältige Situationsanalyse im Rahmen des preispolitischen Entscheidungsprozesses und durch entsprechende Maßnahmen bewältigen. Die positiven Auswirkungen der Ausschöpfung preispolitischer Spielräume zeigen sich für die Museen jedoch nicht nur unmittelbar in der Erhöhung der eigenen Einnahmen - es wird hierdurch auch ein deutliches Signal an potenzielle (private und öffentliche) Finanzierungsgeber gesendet, die künftig jene Museumsbetriebe bevorzugt behandeln werden, die sich bemühen, ihre finanzielle Situation aus eigener Kraft zu verbessern.

\section{Abstract}

\section{Andrea Hausmann, Price strategies for optimizing revenues in cultural institutions}

Cultural institutions; price bundling, price determination; price differentiation; price police; price willingness; self-generated revenues

As economic times are challenging and public funding is dwindling, cultural institutions find themselves in need to explore ways and means to increase self-generated revenues. One way to deal with the situation is to adopt proven price strategies like price differentiation, price variation and price bundling.

\section{Literaturverzeichnis}

Balderjahn, Ingo (1993), Marktreaktionen von Konsumenten, Berlin

Balderjahn, Ingo (2003), Erfassung der Preisbereitschaft, in: Handbuch Preispolitik, hrsg. von Hermann Diller und Andreas Herrmann, Wiesbaden, S. 389-404

Bruhn, Manfred (2003), Sponsoring. Systematische Planung und integrativer Einsatz, 4. Aufl., Wiesbaden

Deutscher Bühnenverein (2004), Theaterstatistik 2002/2003, Köln

Diller, Hermann (2000), Preispolitik, 3. Aufl., Stuttgart

Diller, Hermann (2003), Aufgabenfelder und Ziele der Preispolitik, in: Handbuch Preispolitik, hrsg. von Hermann Diller und Andreas Herrmann, Wiesbaden, S. 3-31

Duda, Alexandra und Andrea Hausmann (2004), Professionelles Management als Erfolgsfaktor im Kultursponsoring, in: Stiftung und Sponsoring, Heft 5, S. 34-37

Fassnacht, Martin (2003), Preisdifferenzierung, in: Handbuch Preispolitik, hrsg. von Hermann Diller und Andreas Herrmann, Wiesbaden, S. 483-502

Giller, Jan (1995), Marketing für Sinfonieorchester, Aachen

Hausmann, Andrea (2001), Besucherorientierung von Museen unter Einsatz des Benchmarking, Bielefeld

Hausmann, Andrea (2005), Theatermarketing: Grundlagen, Methoden und Praxisbeispiele, Stuttgart

Heinrichs, Werner (1997), Kulturpolitik und Kulturfinanzierung, München

Heinze, Thomas (2002), Kultursponsoring, Museumsmarketing, Kulturtourismus, Wiesbaden

Herrmann, Andreas (2003), Relevanz des Preismanagements für den Unternehmenserfolg, in: Handbuch Preispolitik, hrsg. von Hermann Diller und Andreas Herrmann, Wiesbaden, S. 33-45

Institut für Museumskunde (2005), Statistische Gesamterhebung an den Museen der Bundesrepublik Deutschland für das Jahr 2004, Heft 59, Berlin

Institut für Museumskunde (1996), Eintrittspreise von Museen und Ausgabeverhalten der Museumsbesucher, Heft 46, Berlin

Jackson, Ray (1988), A Museum Cost Function, in: Journal of Cultural Economics, Heft 12, S. 41-50

Klein, Armin (1994): Der kommunale Kulturhaushalt: Der Haushaltsplan der Gemeinde, in: Handbuch KulturManagement, hrsg. von Peter Bendixen, Stuttgart, F 1.2, S. 1-30

Klein, Armin (2003), Finanzierung durch Eigenmittel und Umsatzerlöse, in: Handbuch: Erfolgreich Kultur finanzieren, Berlin 
Klein, Armin (2001): Kultur-Marketing. Das Marketingkonzept für Kulturbetriebe, München

Meffert, Heribert und Manfred Bruhn (2003), Dienstleistungsmarketing. Grundlagen, Konzepte und Methoden, 4. Aufl., Wiesbaden

Peacock, Alan T. und Christine Godfrey (1974), The Economics of Museums and Galleries, in: Lloyds Bank Review, Heft 111, S. 17-28

Pechtl, Hans (2005), Preispolitik, Stuttgart

Pommerehne, Werner W. und Bruno S. Frey (1989), Muses and Markets. Explorations in the Economics of the Arts, Oxford

Pommerehne, Werner W. und Bruno S. Frey (1993), Musen und Märkte. Ansätze einer Ökonomik der Kunst, Wiesbaden

Priemer, Verena (2003), Preisbündelung, in: Handbuch Preispolitik, hrsg. von Hermann Diller und Andreas Herrmann, Wiesbaden, S. 503-519

Simon, Hermann (1992), Preismanagement. Analyse, Strategie, Umsetzung, 2. Aufl. Wiesbaden

Söndermann, Michael (2004), Öffentliche Kulturfinanzierung in Deutschland 2003/2004. Ergebnisse aus der Kulturstatistik, in: Jahrbuch für Kulturpolitik 2004, hrsg. von dem Institut für Kulturpolitik der Kulturpolitischen Gesellschaft, Essen, S. 353-375

Statistisches Bundesamt (2004), Kulturfinanzbericht 2003, Wiesbaden

Statistisches Bundesamt (2005), Aktualisierung der Daten zum Kulturfinanzbericht 2003, Wiesbaden

Strachwitz, Rupert Graf (1996), Rechts-, Organisations- und Trägerschaftsformen für Museen und Ausstellungsbetriebe, in: Das Museum als Nonprofit-Organisation, hrsg. von Annette Zimmer, Frankfurt am Main, S. 133-154

Throsby, David und Glenn Withers (1979), The Economics of the Performing Arts, New York

Waetzoldt, Stephan (1974), Das Museum als öffentliche Einrichtung, in: Denkschrift Museen. Zur Lage der Museen in der Bundesrepublik Deutschland und Berlin (West), hrsg. von der Deutschen Forschungsgemeinschaft, Boppard, S. 179-184

Wagner, Bernd (2004), Theaterdebatte - Theaterpolitik, in: hrsg. von Bernd Wagner, Jahrbuch für Kulturpolitik 2004, Thema: Theaterdebatte, Bd. 4, Essen, S. 11-35 